Distribution Category:

LMFBR Structural Materials and Design Engineering (UC-79h)

ANL-77-57

ARGONNE NATIONAL LABORATORY

9700 South Cass Avenue

Argonne, Illinois 60439

AN EFFICIENT NUMERICAL SCHEME BASED ON

THE METHOD OF CHARACTERISTICS APPLICABLE TO

TWO-DIMENSIONAL FLUID TRANSIENTS

by

Yong W. Shin and Richard A. Valentin

Components Technology Division

December 1977

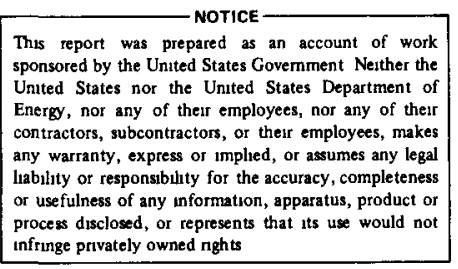




\section{DISCLAIMER}

This report was prepared as an account of work sponsored by an agency of the United States Government. Neither the United States Government nor any agency Thereof, nor any of their employees, makes any warranty, express or implied, or assumes any legal liability or responsibility for the accuracy, completeness, or usefulness of any information, apparatus, product, or process disclosed, or represents that its use would not infringe privately owned rights. Reference herein to any specific commercial product, process, or service by trade name, trademark, manufacturer, or otherwise does not necessarily constitute or imply its endorsement, recommendation, or favoring by the United States Government or any agency thereof. The views and opinions of authors expressed herein do not necessarily state or reflect those of the United States Government or any agency thereof. 


\section{DISCLAIMER}

Portions of this document may be illegible in electronic image products. Images are produced from the best available original document. 
$\bullet$ 
TABLE OF CONTENTS

$\underline{\text { Page }}$

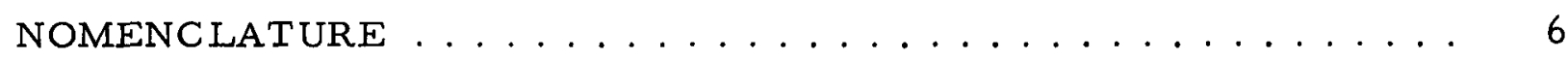

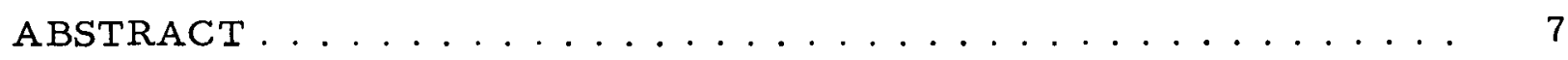

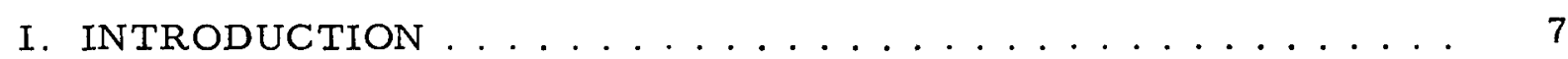

II. BASIC DIFFERENTIAL EQUATIONS. ............. 8

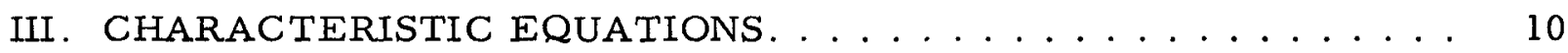

IV. BASIC NUMERICAL SCHEME . . . . . . . . . . . . . . . 12

V. VARIATIONS OF THE BASIC SCHEME ............... 14

VI. SAMPLE PROBLEM CALCULATIONS. . . . . . . . . . . . 17

VII. DISCUSSION AND CONCLUSION. . . . . . . . . . . . . 20

APPENDIX: Conical-flow Solutions for $90^{\circ}$ Corners......... 22

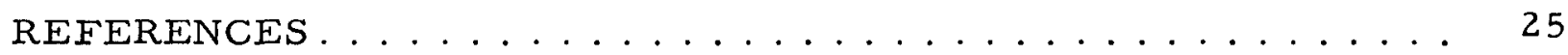




\section{LIST OF FIGURES}

No.

$\underline{\text { Title }}$

$\underline{\text { Page }}$

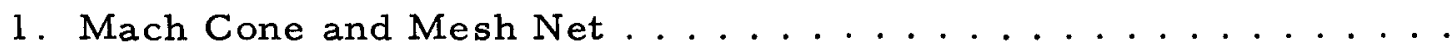

2. Mesh-net Configuration and Mach Cone for a Large-time-step

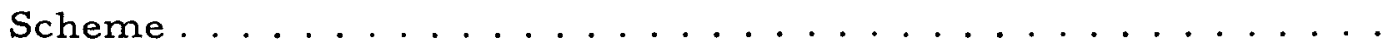

3. Mesh Configurations for $x-y$ Plane Considered as Variations from Basic Method.

4. Integration Paths for Points Next to Boundaries in Large-time-

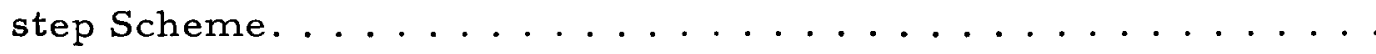

5. Three Sample Problems Considered for Verification of

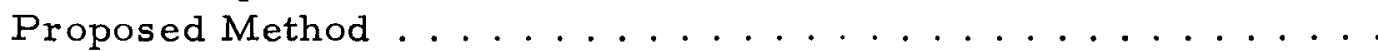

6. Result for Plane-step-pulse Input Compressing around $90^{\circ}$

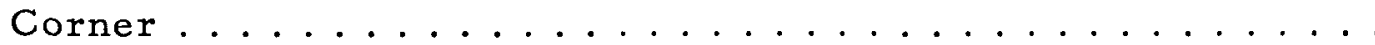

7. Result for Plane-step-pulse Input Expanding into Sudden Area Enlargement

8. One-dimensional Propagation of Step Pulse . . . . . . . . 20

A.1. Compression Problem for $90^{\circ}$ Corner.............. 23

A.2. Expansion Problem for $90^{\circ}$ Corner. . . . . . . . . . . 23

A.3. Exact Solution for Corner Compression . . . . . . . . . . . . 24

A.4. Exact Solution for Corner Expansion . . . . . . . . . . . . . 24

A.5. Individual Wave Fronts for Sudden Area Enlargement . . . . . . . 24 
NOMENCLATURE

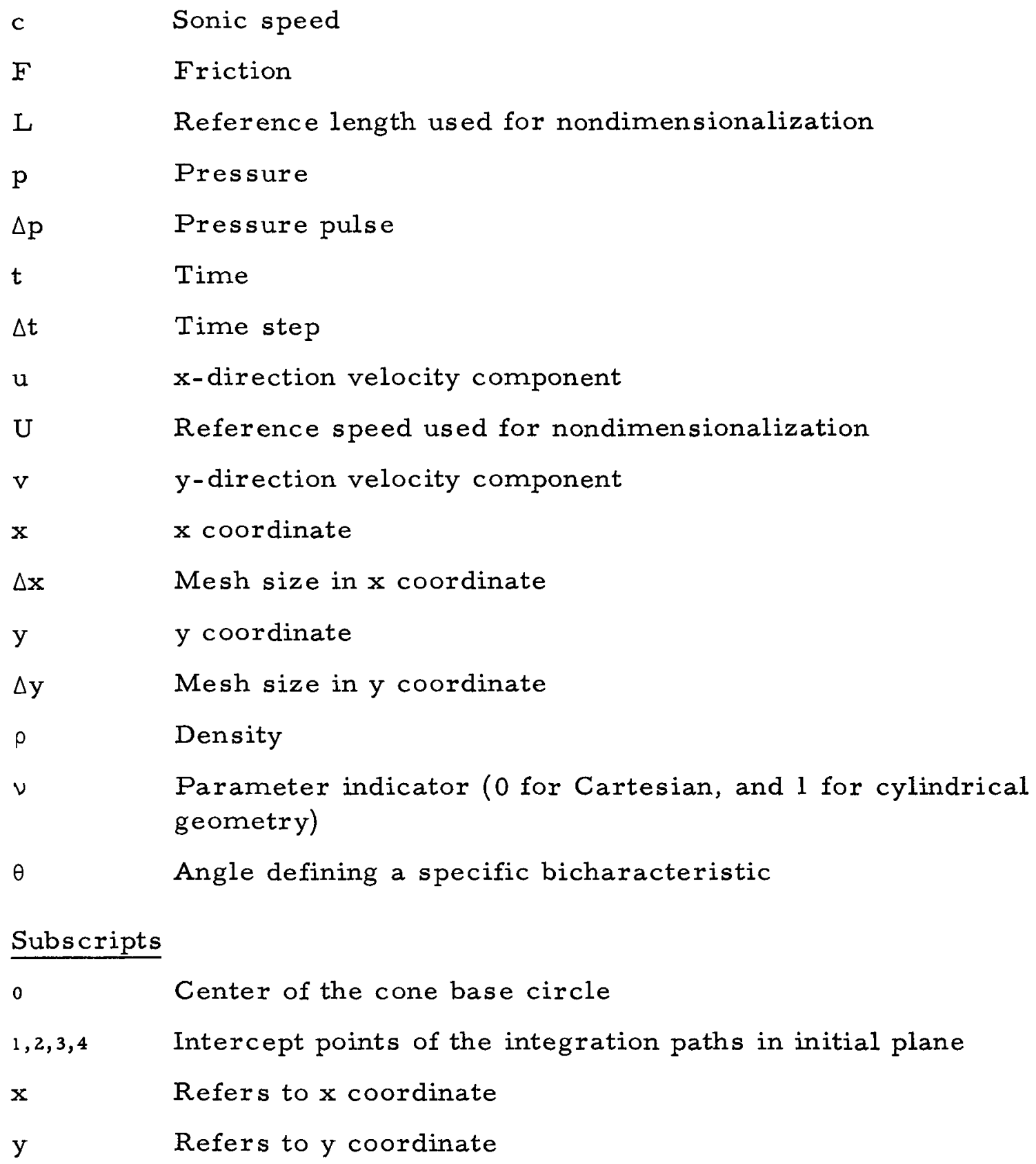




\title{
AN EFFICIENT NUMERICAL SCHEME BASED ON THE METHOD OF CHARACTERISTICS APPLICABLE TO TWO-DIMENSIONAL FLUID TRANSIENTS
}

by

Yong W. Shin and Richard A. Valentin

\begin{abstract}
This report presents two-step explicit numerical procedures based on the method of characteristics. These procedures use four bicharacteristics equally spaced on the Mach cone. The velocity components are first obtained for the entire field using known initial data. Pressure is calculated in the second step in which the advanced velocity data are used. Nowhere in these procedures is an iterative scheme required.

A linearized set of hyperbolic differential equations describing two-dimensional transients in slightly compressible fluid is considered for numerical solution. Detailed schemes are described in which all integration paths (bicharacteristics) remain in the coordinate planes; hence, only simple linear interpolation is required. These schemes allow time steps as large as the entire Courant step. Further, a stable new scheme is discussed in which the allowable time step can actually exceed the Courant time step. The procedure recommended for such computations was chosen after an extensive numerical experimentation that demonstrated its simplicity, efficiency, and accuracy. Exact analytical solutions are constructed and compared with numerical results to demonstrate the accuracy of the recommended scheme.
\end{abstract}

\section{INTRODUCTION}

The method of characteristics is a useful technique for reducing complex differential equations in hyperbolic-type problems to a simpler set of equations suitable for numerical integration. In a general sense, the new set of equations relates conditions along directions in which physical disturbances propagate. Thus a close relationship is maintained between the physical phenomena and the numerical-solution procedure. This results in convenient physical interpretation of the parameters that control numerical stability. In a two-variable case, the systems of partial differential equations are reduced to 
ordinary differential equations, and many well-established techniques are available for obtaining a numerical solution. In problems with more than two independent variables (e.g., multidimensional fluid transient problems), the characteristic equations remain partial differential equations, and numerical solution is more difficult. Nevertheless, numerical techniques have been developed by which these difficulties can be overcome.

Butler ${ }^{1}$ and Richardson ${ }^{2}$ developed a scheme in two space dimensions in which the characteristic partial differential equations are solved numerically just as in the ordinary differential-equation case. The scheme uses four bicharacteristics and one particle path so that gradients in the noncharacteristic directions are eliminated from the difference relations. The resulting relations are then used for the solution of desired flow variables. Shin and Valentin ${ }^{3}$ have used a variation of this scheme for fluid transient analysis. The Von Neumann stability test of their scheme revealed that the time step was restricted to onehalf of the whole Courant step. Sauer ${ }^{4}$ has developed a low-order-accuracy scheme in which multidimensional problems are treated by repeating the scheme of a one-dimensional problem. The integration paths chosen are the lines called nearcharacteristics; gradients in directions other than the nearcharacteristics are evaluated by finite-difference quotients, using initial data. The choice of nearcharacteristics that lie in coordinate planes was applied by Shin and Kot, ${ }^{5}$ who showed that an averaging was required for numerical stability of the two independent solutions obtained from the two coordinate planes. The Von Neumann stability criterion was applied to Sauer's schemes to show the unconditional instability of any of the two schemes if one considers only one coordinate plane. The stability requirement for the third scheme that takes an average of the two unstable solutions was found to be the same as the bicharacteristic scheme. ${ }^{3}$

This report discusses a new scheme that provides improved efficiency and accuracy; the scheme allows the use of large time steps, and hence numerical-dispersion effects are minimized. In the numerical procedure, two calculations are performed for each time-step advancement. In the first calculation, the two velocity components are obtained for the entire field. These advanced velocity components are then used to calculate the new pressures. This scheme, applied to the linearized fluid-hammer equations, uses four bicharacteristics, all lying in coordinate planes. The nearcharacteristic lines collapse onto the bicharacteristics in this linearized problem.

\section{BASIC DIFFERENTIAL EQUATIONS}

The basic equations considered for a generally compressible flow are the conservation equations of mass, momentum, and energy. In many transient problems, however, it is sufficient to consider the fluid as only slightly 
compressible. This enables an approximation (the so-called fluid-hammer approximation) in which the energy equation is replaced with a simple isentropic relation. The resulting differential equations, in two dimensions, are (with the is entropic relation in differential form) as follows.

Continuity:

$\frac{\partial \rho}{\partial t}+u \frac{\partial \rho}{\partial x}+v \frac{\partial \rho}{\partial y}+\rho\left(\frac{\partial u}{\partial x}+\frac{\partial v}{\partial y}+v \frac{v}{y}\right)=0$.

Momentum:

$$
\frac{\partial u}{\partial t}+u \frac{\partial u}{\partial x}+v \frac{\partial u}{\partial y}+\frac{1}{\rho} \frac{\partial p}{\partial x}+F_{x}=0
$$

and

$$
\frac{\partial v}{\partial t}+u \frac{\partial v}{\partial x}+v \frac{\partial v}{\partial y}+\frac{1}{\rho} \frac{\partial p}{\partial y}+F_{y}=0
$$

Isentropic relation:

$$
d p=c^{2} d p .
$$

Equation 1 is combined with Eq. 4 to eliminate the density change:

$$
\frac{\partial p}{\partial t}+u \frac{\partial p}{\partial x}+v \frac{\partial p}{\partial y}+\rho c^{2}\left(\frac{\partial u}{\partial x}+\frac{\partial v}{\partial y}+v \frac{v}{y}\right)=0 .
$$

Equations 2, 3, and 5 form a set of three equations with three unknowns: the pressure $p$, and the two velocity components $u$ and $v$. Typically, the sonic speed in any liquid is large; hence, for any given pressure change the corresponding change in density is small, as indicated in Eq. 4. It is thus reasonable to assume constant density in these equations.

In most cases, the fluid velocities in liquids are small, compared to the sonic speeds. For example, in still water at room temperature, a fluid particle velocity of about $4.57 \mathrm{~m} / \mathrm{s}(15 \mathrm{ft} / \mathrm{s}$ ) is developed by a pressure pulse of $6.89 \mathrm{MPa}$ (1000 psi). Since the sonic speed in water is $1487 \mathrm{~m} / \mathrm{s}(4880 \mathrm{ft} / \mathrm{s})$, the particle velocity is only $0.3 \%$ of the sonic speed. Thus, for pressure pulses of this order of magnitude, it is reasonable to linearize the above equations and obtain

$$
\frac{\partial u}{\partial t}+\frac{\partial p}{\partial x}+F_{x}=0
$$




$$
\frac{\partial v}{\partial t}+\frac{\partial p}{\partial y}+F_{y}=0
$$

and

$$
\frac{\partial p}{\partial t}+\frac{\partial u}{\partial x}+\frac{\partial v}{\partial y}+\nu \frac{v}{y}=0
$$

These equations have been written in dimensionless form, using the following nondimensionalization:

$$
\begin{aligned}
(\mathrm{u}, \mathrm{v}) & =\left(\mathrm{u}^{*}, \mathrm{v}^{*}\right) / \mathrm{U} \\
\mathrm{p} & =\mathrm{p}^{*} / \mathrm{pcU}, \\
\left(\mathrm{F}_{\mathrm{x}}, \mathrm{F}_{\mathrm{y}}\right) & =\left(\mathrm{F}_{\mathrm{x}}^{*}, \mathrm{~F}_{\mathrm{y}}^{*}\right) \mathrm{L} / \mathrm{Uc}, \\
(\mathrm{x}, \mathrm{y}) & =\left(\mathrm{x}^{*}, \mathrm{y}^{*}\right) / \mathrm{L}
\end{aligned}
$$

and

$$
\mathrm{t}=\mathrm{ct} * / \mathrm{L} \text {, }
$$

where the asterisks identify the dimensional variables. The reference velocity $U$ can be obtained in terms of a reference pressure pulse $\Delta p$ as

$$
\mathrm{U}=\frac{\Delta \mathrm{p}}{\rho \mathrm{c}}
$$

\section{CHARACTERISTIC EQUATIONS}

The hyperbolic system of linearized partial differential equations 6-8 are linearly combined to yield a new set of equations in a characteristic form. This new set, unlike the case of two-variable problems, includes infinitely many equations from which a choice is made for a suitable solution procedure. As derived in detail in Ref. 3, the set of characteristic equations are written parametrically as

$$
\begin{aligned}
\frac{d p}{d t}- & \cos \theta \frac{d u}{d t}-\sin \theta \frac{d v}{d t}+\sin ^{2} \theta\left(\frac{\partial u}{\partial x}+\nu \frac{v}{y}\right)+\cos ^{2} \theta\left(\frac{\partial v}{\partial y}+v \frac{v}{y}\right) \\
& -\sin \theta \cos \theta\left(\frac{\partial u}{\partial y}+\frac{\partial v}{\partial x}\right)=\cos \theta \cdot F_{x}+\sin \theta \cdot F_{y},
\end{aligned}
$$


where

$$
\frac{d}{d t}=\frac{\partial}{\partial t}-\cos \theta \frac{\partial}{\partial x}-\sin \theta \frac{\partial}{\partial y}
$$

is a derivative along a bicharacteristic identified by a particular value of $\theta$ between 0 and $2 \pi$. In Fig. 1, a local Mach cone is shown with its vertex at $P$ and the initial data in the $x-y$ plane. With $\theta$ as defined in Fig. 1, d/dt of Eq. 12 is easily identified as a derivative along a bicharacteristic--a line connecting a point on the base circle with point $P$.

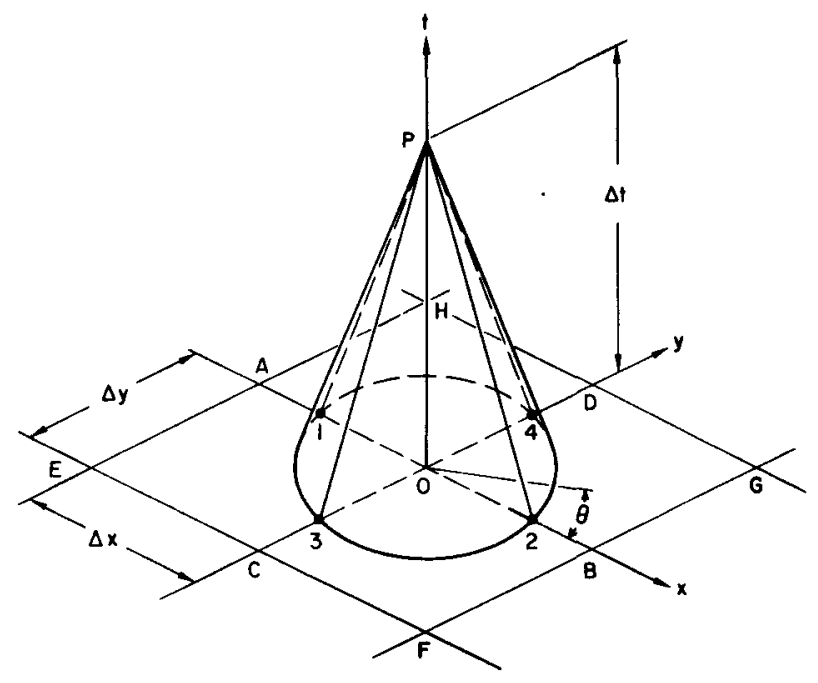

Fig. 1

Mach Cone and Mesh Net. Bicharacteristics 1P, $2 \mathrm{P}, 3 \mathrm{P}$, and $4 \mathrm{P}$ are integration paths. ANL Neg. No. 113-77-249.

A choice of four equations is made by selecting $\theta=0, \pi / 2, \pi$, and $3 \pi / 2$. Two of the four spatial derivatives, $\partial u / \partial y$ and $\partial v / \partial x$, are eliminated in this procedure, and the resulting equations are written as difference relations:

$$
\begin{aligned}
& p-p_{1}+\left(u-u_{1}\right)+\Delta t\left(\frac{\partial v}{\partial y}+\nu \frac{v}{y}\right)=-\Delta t\left(F_{x_{1}}\right)_{1}, \\
& p-p_{2}-\left(u-u_{2}\right)+\Delta t\left(\frac{\partial v}{\partial y}+\nu \frac{v}{y}\right)=\Delta t\left(F_{x}\right)_{2} \\
& p-p_{3}+\left(v-v_{3}\right)+\Delta t\left(\frac{\partial u}{\partial x}+v \frac{v}{y}\right)=-\Delta t\left(F_{y}\right)_{3},
\end{aligned}
$$

and

$$
p-p_{4}-\left(v-v_{4}\right)+\Delta t\left(\frac{\partial u}{\partial x}+\nu \frac{v}{y}\right)=\Delta t\left(F_{y}\right)_{4}
$$

Where appearing, variables and derivatives without subscripts refer to the advanced point $P$, and subscripts 1-4 represent base points of the four 
bicharacteristics (see Fig. 1). The frictional terms are evaluated using the initial data, and the divergence terms $\partial u / \partial x, \partial v / \partial y$, and $v / y$ are placed at the new point $P$.

Equations 13 are the basic difference relations used to construct the computational schemes discussed in this report. Variables at other than the mesh points in the initial plane are numerically evaluated by linear interpolation, using neighboring mesh points. For example, $u_{3}$ in Fig. 1 is evaluated using $\mathrm{u}_{\mathrm{C}}$ and $\mathrm{u}_{0}$ :

$$
u_{3}=r u_{C}+(1-r) u_{0}
$$

where

$$
r=\overline{\mathrm{O} 3} / \overline{\mathrm{OC}}
$$

\section{BASIC NUMERICAL SCHEME}

Equations 9 allow us to formulate a procedure whereby the velocity components can be obtained for the entire field without a simultaneous solution for the pressure. Subtraction of Eq. 13b from Eq. 13 a yields a solution for $u$, and subtraction of Eq. $13 \mathrm{~d}$ from Eq. 13c provides a solution for $\mathrm{v}$ :

$$
u=\frac{1}{2}\left\{u_{1}+u_{2}+p_{1}-p_{2}-\Delta t\left[\left(F_{x}\right)_{1}+\left(F_{x}\right)_{2}\right]\right\}
$$

and

$$
v=\frac{1}{2}\left\{v_{3}+v_{4}+p_{3}-p_{4}-\Delta t\left[\left(F_{y}\right)_{3}+\left(F_{y}\right)_{4}\right]\right\}
$$

These velocity calculations are performed for the entire field first. Then new velocity values are used to construct the procedure for pressure calculation. Addition of Eqs. $13 \mathrm{a}$ and $13 \mathrm{~b}$ yields

$$
p=\frac{1}{2}\left\{p_{1}+p_{2}+u_{1}-u_{2}-\Delta t\left[\left(F_{x}\right)_{1}-\left(F_{x}\right)_{2}\right]\right\}-\Delta t\left(\frac{\partial v}{\partial y}+v \frac{v}{y}\right)
$$

An equally valid choice is the use of Eqs. $13 \mathrm{c}$ and 13d, which results in

$$
p=\frac{1}{2}\left\{p_{3}+p_{4}+v_{3}-v_{4}-\Delta t\left[\left(F_{y}\right)_{3}-\left(F_{y}\right)_{4}\right]\right\}-\Delta t\left(\frac{\partial u}{\partial x}+v \frac{v}{y}\right)
$$

The derivatives of Eqs. 16 and 17 can be evaluated by difference quotients, using field values of $u$ and $v$ at the adjacent mesh points. High-order central differences are employed for interior mesh points. At the boundaries, however, lower-order sided differences are used. 
Equations 14-16--or Eq. 17 in place of Eq. 16 as an alternative-constitute the basic scheme for interior mesh points where all four bicharacteristics are available for use. At the boundary meshes, however, some bicharacteristics fall outside the computational domain. In these cases, appropriate boundary conditions provide needed information on velocities; for example, at a rigid x-boundary, $u=0$, and at a $y$-boundary, $v=0$. Nonzero velocities also can be specified if such condition exists, as in the fluid-structure interaction problem. The pressure, however, still must be calculated.

Pressure calculation at boundaries is performed somewhat differently than at interior points. Either Eq. 16 or 17 is used first, whichever is applicable; for example, at an x-boundary, Eq. 17 is used. A second calculation is then made and used to form an algebraic average with the first calculation. This second calculation considers the available bicharacteristic relation (one of Eqs. 13 normal to the boundary. For example, at an $x$-boundary, $x=0$, where $x<0$ is external to the domain, Eq. $13 b$ is used. In this cause, u appearing in the equation will represent the wall velocity.

At a corner point where both boundaries exist, both Eqs. 14 and 15 are replaced by wall conditions; i.e., $u=0$ and $v=0$. Here, neither of the pressure equations, Eqs. 16 and 17, is applicable. Independent pressure calculations are made from the two available equations of Eqs. 13, and the results are then averaged. At the nonboundary axial points for a cylindrical geometry, $y=0$, the term $v / y$ is not defined, and the term is replaced by $\partial v / \partial y$.

The numerical stability of the foregoing scheme is governed by the Courant-Friedrichs-Lewy ${ }^{6}$ (CFL) condition that applies to all hyperbolic systems: The domain of dependence of the difference system must contain the domain of dependence of the differential system. With reference to Fig. 1, the domain of dependence of the differential system is the base circle of the Mach cone, and the difference domain is considered to be the rectangle EFGH. Hence, the CFL condition requires that the base circle should lie inside the rectangle EFGH, which implies that

$$
\frac{\Delta t}{\Delta x} \leq 1
$$

The CFL condition is not sufficient for stability. A sufficient condition can be found by Fourier analysis, as was done in Ref. 3. Here, however, a series of numerical experiments was performed as an alternative method. The problem chosen for this purpose was a rectangular domain with $5 \times 10 \mathrm{mesh}$ points in which an initial pressure discontinuity was given to induce twodimensional flow development from an initially stagnant condition. Calculations were continued for 250 time steps, and the qualitative features of the solution

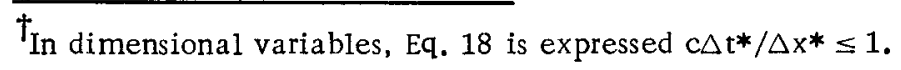


were used to define numerical stability for a given time step. The result showed clear stability when the time step was chosen as

$$
\frac{\Delta \mathrm{t}}{\Delta \mathrm{x}} \leq 4 / 5
$$

This time step is a significant increase from earlier schemes in which the requirement was $1 / 2$. Equation 19 applies to both Cartesian and cylindrical geometry.

\section{VARIATIONS OF THE BASIC SCHEME}

Many variations of the basic scheme were formulated and examined for accuracy and stability. These all used the same velocity equations, Eqs. 14 and 15 , as the basic scheme of the previous section. However, the pressure calculations were done differently. In the first scheme, the pressures were calculated by taking an average of two independent calculations, using Eqs. 16 and 17, respectively, for all points (interior and boundary). It differs from the basic scheme discussed in the previous section in that the basic scheme employs the pressure averaging for the boundary nodes only. The pressure averaging for all nodes allowed the first scheme for a slightly greater time step with generally a comparable accuracy to the basic scheme. Nevertheless, the basic scheme is preferred, since it reduces identically to the one-dimensional method of characteristics scheme and yields more accurate results when onedimensional waves are encountered.

This property of the basic scheme is considered important to applications involving long geometries where, for points far from the disturbance source, the waves are essentially one-dimensional and the accuracy is important. The time step allowed for Cartesian geometry was as large as a whole Courant step, i.e., $\Delta t / \Delta x=1$. For a cylindrical problem, this scheme required a slightly reduced time step, $\Delta t / \Delta x=0.9$. In the second scheme, one pressure equation, Eq. 16, was used for every mesh point. This resulted in the limitation $\Delta t / \Delta x=$ 3/4. Due to the smaller time step, this scheme exhibited a slightly greater dispersion effect than the basic scheme.

Other schemes examined included different ways of treating spatial derivatives appearing in the pressure equations. These derivatives were placed in the current time plane instead of the advanced plane as in the basic scheme and in the schemes discussed above. (The spatial derivatives were formed with known current time values.) These schemes further reduced the allowable time step and increased dispersion errors. Also examined were schemes involving different combinations of pressure calculations in the interior as well as on boundaries, and with the spatial derivatives placed in the advanced time plane. These schemes were either comparable or inferior to the basic scheme in both stability and accuracy. 
The basic scheme and an earlier scheme ${ }^{3}$ were applied to a different mesh net in $t-x-y$ space involving a time step exceeding the Courant number unity. In this case, the characteristics project over more than one mesh. The case of square meshes is shown in Fig. 2. The base-point values (values at points $1,2,3$, and 4) were evaluated by linear interpolation, using adjacent mesh-point values. As such, the necessary CFL condition is satisfied, since the numerical domain encloses the differential domain. Nevertheless, many of the mesh configurations examined proved to be unconditionally unstable. Two of the most important configurations are shown in Figs. $3 \mathrm{~b}$ and $3 \mathrm{c}$. These would be desirable since the dispersion errors would be minimal and such configurations would be suitable for problems with major extension in one coordinate direction (such as a very long or very short cylinder). Unfortunately, no stable schemes were found for these configurations.

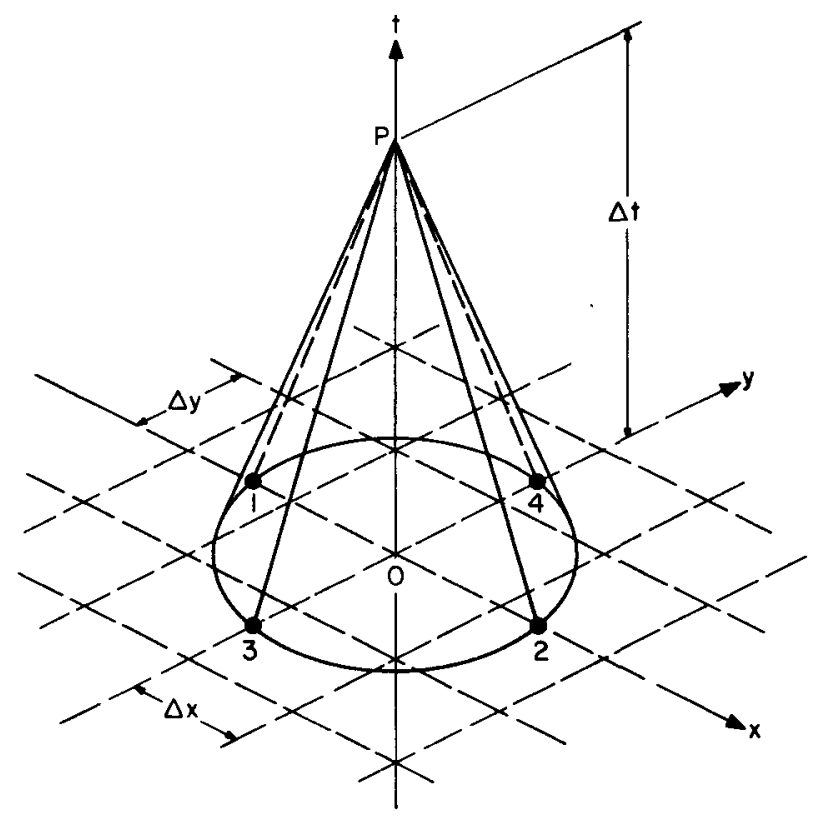

Fig. 2. Mesh-net Configuration and Mach Cone for a Large-time-step Scheme. (Time step is greater than CFL step.) ANL Neg. No. $113-77-547$.

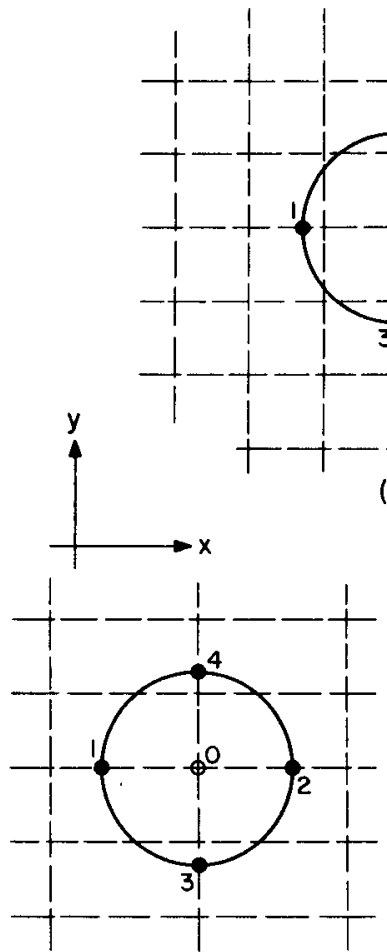

(b)

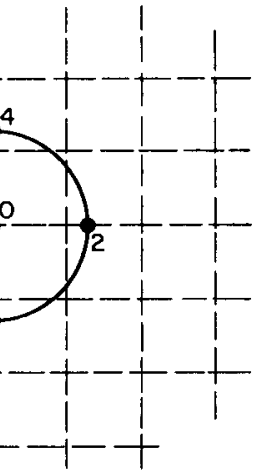

(a)
Fig. 3. Mesh Configurations for $x-y$ Plane Considered as Variations from Basic Method. ANL Neg. No. 113-77-545.

The configuration shown in Fig. 3a, however, was shown to be stable using the basic scheme discussed in the previous section. (The earlier scheme showed unconditional instability in this case.) The stability requirement was found to be

$$
1 \leq \frac{\Delta t}{\Delta x} \leq 1.3
$$


in both Cartesian and cylindrical coordinates. The characteristic equations, Eqs. 13, apply to this case in general; however, special calculations must be made for mesh points next to the boundary. In this case, one of the four characteristics changes its direction (two in the case of a corner point); hence, the integration path involves two different characteristics. Figure 4 indicates the four possible cases in which $P$ is the mesh point one mesh away from boundaries. Consider the first case of the boundary, $x=0$, in which case the characteristic equation along the path $\overline{I^{\prime} P}$ requires a special treatment. Path $\overline{I^{\prime} P}$ is the characteristic corresponding to a $\theta$ value of $3 \pi / 2$ (refer to Fig. 1 ), and, letting the associated time step be $\Delta t_{1}$, the characteristic equation can be written

$$
p-p_{1}+\left(u-u_{1}^{\prime}\right)+\Delta t_{1}\left(\frac{\partial v}{\partial y}+v \frac{v}{y}\right)=-\Delta t_{1} \cdot\left(F_{x}\right)_{1} \cdot
$$

For $\overline{1 \Gamma^{\prime}}$, the equation for $\theta=\pi / 2$ with time step $\Delta t_{2}$ is

$$
p_{1},-p_{1}-\left(u_{1},-u_{1}\right)+\Delta t_{2}\left(\frac{\partial v}{\partial y}+v \frac{v}{y}\right)_{1^{\prime}}=\Delta t_{2} \cdot\left(F_{x}\right)_{1}
$$

Adding these two equations and using the same approximations for the inhomogeneous terms as in Eqs. 13, we obtain

$$
p-p_{1}+\left(u-2 u_{1}{ }^{\prime}+u_{1}\right)+\Delta t\left(\frac{\partial v}{\partial y}+v \frac{v}{y}\right)=-\Delta t\left(F_{x}\right)_{1}
$$

Here, $\Delta t=\Delta t_{1}+\Delta t_{2}$ was used. The form of this equation differs only slightly from the corresponding Eq. 13a: $u_{1}$ ' is the wall normal velocity. For other
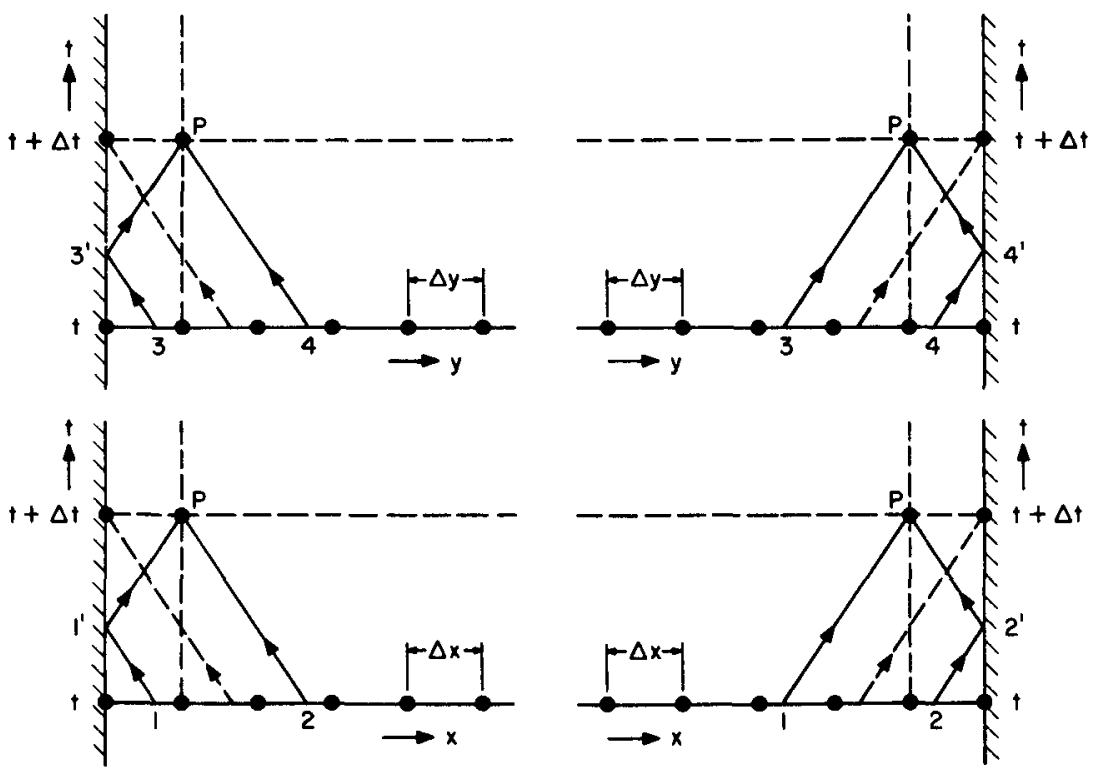

Fig. 4. Integration Paths for Points Next to Boundaries in Largetime-step Scheme. ANL Neg. No. 113-77-543. 
walls, different characteristics require special treatment. For the wall y $=0$,

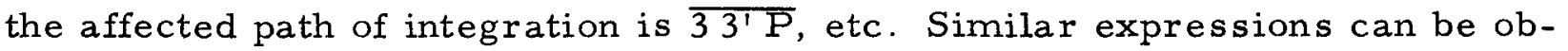
tained for the remaining wall cases as follows:

$$
\begin{aligned}
& p-p_{2^{\prime}}-\left(u-2 u_{2^{\prime}}+u_{2}\right)+\Delta t\left(\frac{\partial v}{\partial y}+v \frac{v}{y}\right)=\Delta t\left(F_{x}\right)_{2^{\prime}} \\
& p-p_{3}+\left(v-2 v_{3^{\prime}}+v_{3}\right)+\Delta t\left(\frac{\partial u}{\partial x}+v \frac{v}{y}\right)=-\Delta t\left(F_{y}\right)_{3^{\prime}}
\end{aligned}
$$

and

$$
p-p_{4}-\left(v-2 v_{4}+v_{4}\right)+\Delta t\left(\frac{\partial u}{\partial x}+v \frac{v}{y}\right)=\Delta t\left(F_{y}\right)_{4}
$$

For mesh points near corners, two of Eqs. 21 must be used instead of the corresponding single equation from those listed in Eqs. 13. Proper combinations of Eqs. 13 and 21 enable construction of a complete solution scheme, which is essentially the same as the basic method described in Section IV: Velocity calculations are performed first for the entire field, and the results are used to form velocity gradients needed for the pressure calculations.

The velocity gradients use the nearest neighbor points in the difference quotients. Pressure calculations use $\mathrm{x}$-direction characteristics only for the interior mesh points; averaging is used for boundary points.

\section{SAMPLE PROBLEM CALCULATIONS}

Simple sample problems were considered to verify the numerical technique by comparison with exact analytical solutions. Analytical solutions were generated, using the method discussed by Keller and Blank. ${ }^{7}$ This involves transformation of the wave equation into a Laplace equation, using Busemann's conical-flow technique. ${ }^{8}$ This method, combined with a conformal mapping, was used to solve for a step pressure pulse traversing a $90^{\circ}$ corner. The resultant analytical solution forms the basis for verifying the numerical method discussed earlier. Details of the analytical solutions are given in the appendix.

Three sample problems were chosen and are shown in Fig. 5. These problems were selected to test as many features of the numerical technique as possible, while remaining simple enough to be amenable to exact solution. The first sample problem, shown in Fig. 5a, involves two-dimensional (Cartesian) wave propagation of a step pulse around a $90^{\circ}$ corner. The diffracted wave from the corner interacts with both the undisturbed and the reflected wave front. Initially, a high pressure $\left(p_{2}\right)$ is placed one mesh from the corner so that the flow accelerates into the low-pressure region $\left(p_{1}\right)$. 


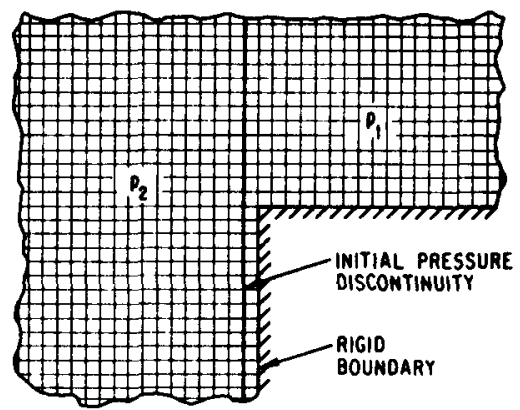

(0) TEST CASE 1 (COMPRESSION ON $90^{\circ}$ WEDGE)

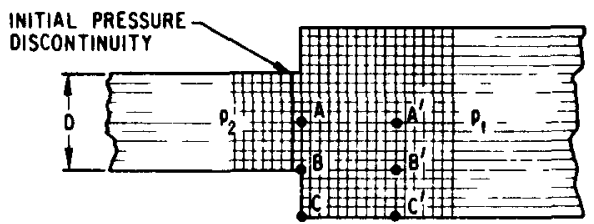

(0) TEST CASE 2 (DECOMPRESSION INTO SUDOEN EMLARGEMENT)

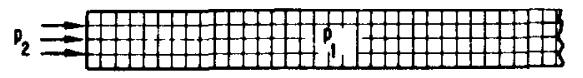

(c) TESt CaSE 3 ( Propagation Of PLAME PRESSURE pULSE)

Fig. 5. Three Sample Problems Considered for Verification of Proposed Method. ANL Neg. No. 113-77-358 Rev. 1.
In the second sample problem (Fig. 5b), there is a sudden area enlargement, again in Cartesian geometry, and two features not contained in the first example are involved. In this case, the diffracted waves originating from the two corners interact with each other. This problem also involves diffracted waves being reflected from flat surfaces. Here again, the transient is developed from an initial unbalance of pressure. The area ratio considered is 1 to 2 . The analytical solution for this sample problem was obtained by superposition of simpler problems, as described in the appendix.

The third sample problem is onedimensional and was intended to test numerical-dispersion effects for onedimensional waves. Again, a step pulse was used and wave shapes were examined as the wave front propagated down the pipe.

Figures 6 and 7 compare the numerical calculations and analytical solutions for the first and second sample problems, respectively. Figure 6 plots pressure distribution on the wedge surfaces up to the diffraction wave front.

Fig. 6

Result for Plane-step-pulse Input Compressing around $90^{\circ}$ Corner (Test Case 1). ANL Neg. No. 113-77-356 Rev. 1.

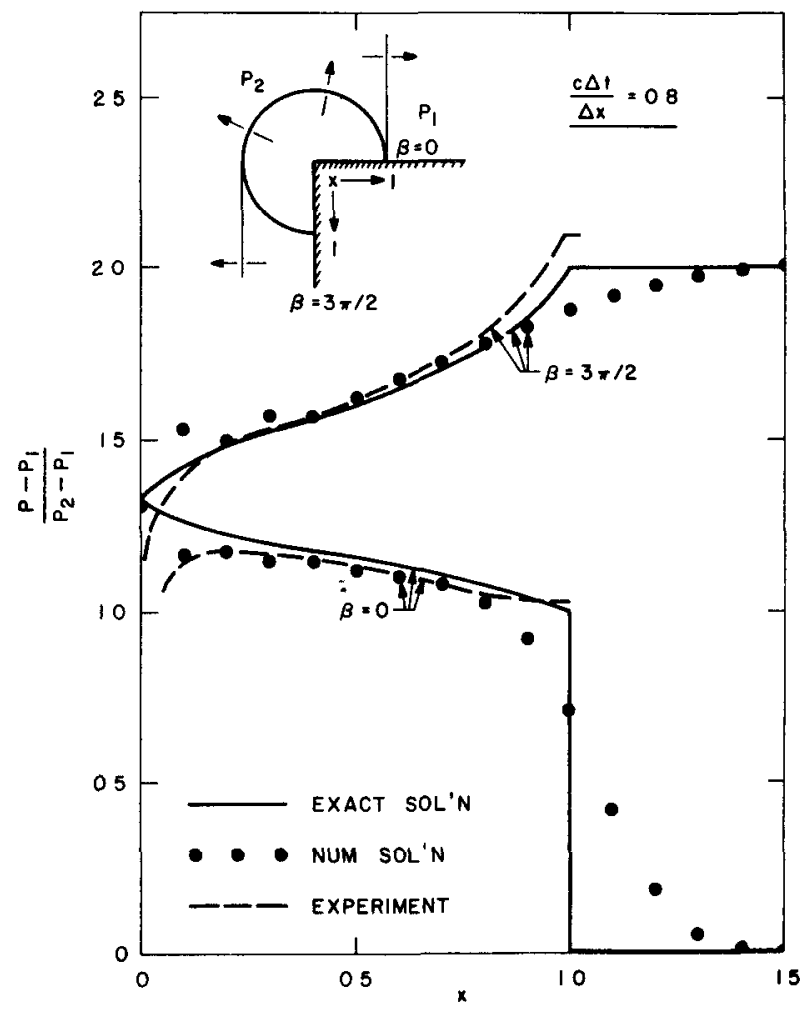



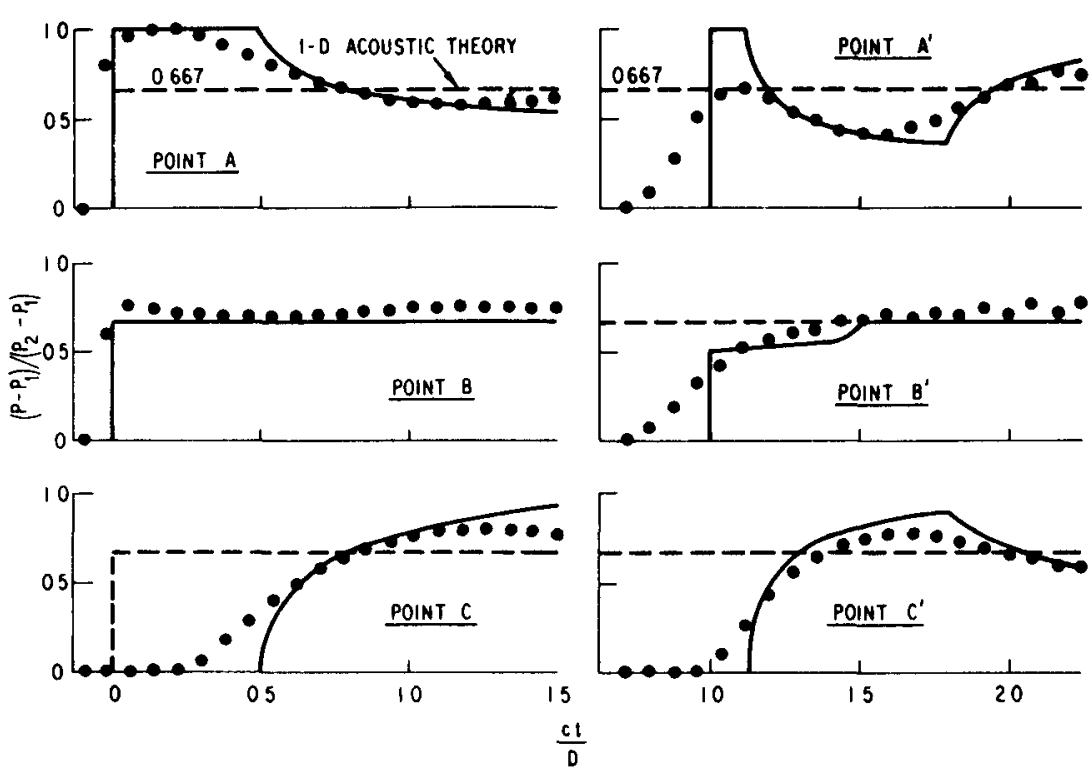

Fig. 7. Result for Plane-step-pulse Input Expanding into Sudden Area Enlargement (Test Case 2). ANL Neg. No. 113-77-355.

Generally, the agreement is good. At the discontinuity front $(\theta=0$ in Fig. 6), some dispersiveness of the numerical scheme can be seen. Experimental data obtained by White and Bleakney ${ }^{9}$ are also plotted for comparison. The discrepancy between the experimental data and the analytical or the numerical solution is due to the vortex phenomenon around the corner. Viscous effects associated with the corner vortex are neglected in the analytical and numerical treatments. White and Bleakney used a $12 \%$ excess pressure, $p_{2} / p_{1}=1.12$, which is low enough so that linear behavior could be expected.

Results for the sudden-enlargement problem (second sample problem) are given in Fig. 7. Here again, the agreement is good, except for some numerical spreading near sharp gradient changes. In particular, at point $A^{\prime}$ (see Fig. 5b for its location), the loss in the initial peak pressure (short duration) is appreciable, although the general pressure history is in good agreement. Early arrivals of the disturbance pulses are also evident and can be attributed to numerical-dispersion effects.

Figure 8 depicts the results for the one-dimensional step pulse of the last sample problem. The waveform, as it travels down the pipe, is progressively dispersed. (The effects are cumulative.) Results obtained using an earlier technique ${ }^{3}$ are plotted in Fig. $8 \mathrm{~b}$ for comparison. The numerical smearing is much less in the present scheme (see Fig. 8a) than in the earlier results. This improvement is important in problems that involve a long extension in one spatial coordinate compared to the other. The current analysis mimimizes cumulative dispersion effects, which could otherwise result in unconservative estimates. Note also the difference in the required number of computation steps for the input pulse to reach a certain point. This results from the different size of time steps required for numerical stability of the two methods. 


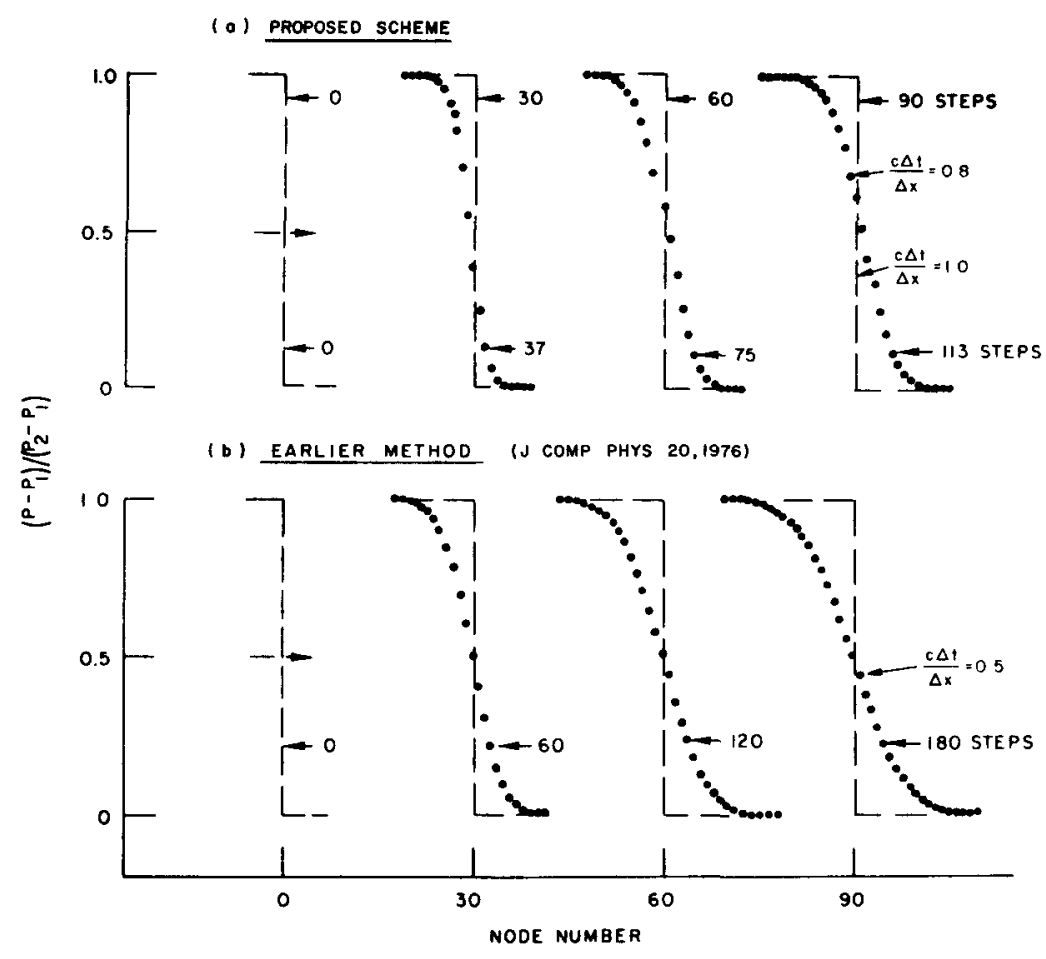

Fig. 8. One-dimensional Propagation of Step Pulse (Test Case 3). ANL Neg. No. 113-77-357.

The three sample-problem calculations verify many basic features of the proposed method. Besides the general interior and rigid-boundary point calculations, the numerical scheme can calculate diffraction waves and their interactions with reflected and undisturbed plane waves. The diffraction of a curved wave front has not been considered since the analytical solution is not available, and the construction method used for the other exact solutions is limited to a plane discontinuity. The proposed scheme was found stable for the mesh configuration described in Fig. 3a, as discussed earlier. However, the practical importance of this case was not evident; hence, no effort was made to verify its numerical stability. The basic scheme was used in all the numerical calculations shown for which the maximum allowable time step $\Delta \mathrm{t} / \Delta \mathrm{x}=0.8$ was used.

\section{DISCUSSION AND CONCLUSION}

This report has described an efficient numerical method based on the bicharacteristic formulation as applied to the linearized set of fluid-hammer equations. The choice of the integration paths from the infinitely available paths resulted in intercepts at mesh lines so that only linear interpolation was required in the initial plane. Because of the linearized characteristics, the intercepts of the integration paths with the initial plane can be determined at the start of the calculation. Also, because there are many available choices 
for integration paths, many schemes can be devised, using different combinations of the chosen bicharacteristics or explicit or implicit treatment of the inhomogeneous terms of the characteristic equations. Several such schemes were examined and their accuracies tested. As a result of extensive numerical experimentation, we concluded that the numerical technique discussed here is likely to be the simplest, most accurate, and computationally efficient.

Although general accuracy of the proposed method is demonstrated through sample problem calculations, the scheme unfortunately remains dispersive. This is in contrast with the one-dimensional method in which the scheme is essentially nondispersive. When only plane waves are encountered, the proposed method reduces indentically to the one-dimensional method. We have found, although it is not presented in this report, that by use of the maximum allowable time step $\left(\Delta t * / \Delta x^{*}=1\right)$, the method can calculate plane step pulses propagating with no dispersive smoothing effects. Therefore, we recommend that the two-dimensional scheme be used only in regions where detailed local effects are of interest and, whenever possible, the remainder of the domain be calculated by a one-dimensional scheme. This can be done by providing appropriate approximations at the interface to connect the two schemes. For best accuracy, the maximum allowable time step should always be used.

Applicability of the method to a nonsquare mesh system was also examined. This is an important option for problems with a domain relatively long in one direction, e.g., a long cylinder. Unfortunately, the scheme proved numerically unstable for all meaningful time steps. For these problems, which are in essence one-dimensional, the exclusive use of a two-dimensional scheme can result in an excessive dispersive error. The size of the error will, of course, depend on the waveform, especially for the long-time response. Here again, substitution of a one-dimensional scheme in certain parts of the domain can enhance the overall accuracy of the result.

Although the method has been presented here in terms of fluid-transient equations, it should be equally applicable, with small modifications, to the more general case of compressible flows. Modifications would include the use of a full nonlinear formulation, two-dimensional interpolation, and the inclusion of variable characteristics that probably would require an iterative solution procedure. Dispersiveness will still exist; nevertheless, the method should provide an accurate computational tool for a large class of compressible flow transients when used in conjunction with a one-dimensional scheme to control numerical dispersion. 


\section{APPENDIX}

Conical-flow Solutions for $90^{\circ}$ Corners

Conical-flow solutions used to construct the solution for a sudden area enlargement are outlined below. The basic solution procedure is that given by Keller and Blank. ${ }^{7}$ The wave equation* is obtained by combining Eqs. 6 and 7 and neglecting friction:

$$
\frac{\partial^{2} p}{\partial x^{2}}+\frac{\partial^{2} p}{\partial y^{2}}-\frac{1}{c^{2}} \frac{\partial^{2} p}{\partial t^{2}}=0
$$

The boundary condition used is

$$
\frac{\partial p}{\partial n}=0
$$

where $\mathrm{n}$ is the coordinate normal to the boundary. This results from Eq. 6, for example, with $u=0$ at the $x$ boundary.

Following Busemann's conical-flow method, Eq. A.l is rewritten in terms of new variables (similarity variables) and becomes a Laplace's equation in polar coordinates in terms of the similarity variables:

$$
\gamma \frac{\partial}{\partial \gamma}\left(\gamma \frac{\partial p}{\partial \gamma}\right)+\frac{\partial^{2} p}{\partial \alpha^{2}}=0,
$$

where

and

$$
\left.\begin{array}{l}
\gamma=\left(\frac{q-1}{q+1}\right)^{1 / 2}, \\
q=\frac{c t}{\left[c^{2} t^{2}-\left(x^{2}+y^{2}\right)\right]^{1 / 2}},
\end{array}\right\}
$$

$$
\alpha=\tan ^{-1} \mathrm{y} / \mathrm{x}
$$

The problem is thus to solve Eq. A.3 for a $90^{\circ}$ wedge with vanishing normal derivatives at the wedge surfaces. This can be reduced to a unit-circle problem by mapping the exterior of the wedge into the interior of the unit circle.

The closed-form solutions for a step-pulse compressing and expanding around a $90^{\circ}$ corner are obtained as follows:

*The variables in this and subsequent equations represent dimensional quantities. 
For the compressing step pulse,

$$
\frac{p-p_{1}}{p_{2}-p_{1}}=1+\frac{1}{\pi} \arctan \frac{\left(1-\ell^{4 / 3}\right) \cos \frac{\pi}{6}}{\left(1+\ell^{4 / 3}\right) \sin \frac{\pi}{6}+2 l^{2 / 3} \cos \frac{2 \beta}{3}},
$$

and for the expanding step pulse,

$$
\frac{p-p_{1}}{p_{2}-p_{1}}=\frac{1}{\pi} \arctan \frac{\left(1-\ell^{4 / 3}\right) \sin \frac{\pi}{3}}{-\left(1+\ell^{4 / 3}\right) \cos \frac{\pi}{3}+2 \ell^{2 / 3} \cos \frac{2 \beta}{3}},
$$

where

$$
l=\frac{R}{c t+\left(c^{2} t^{2}-R^{2}\right)^{1 / 2}}
$$

$R$ is the distance from the corner, and $\beta$ is the angle measured from one of the wedge surfaces, as indicated in Figs. A. 1 and A.2. The solutions as expressed in Eqs. A. 5 and A. 6 are plotted in the $(\ell, \beta)$ plane in Figs. A. 3 and A. 4 . The solution for the compression corner is the same as that obtained by Keller. ${ }^{10}$

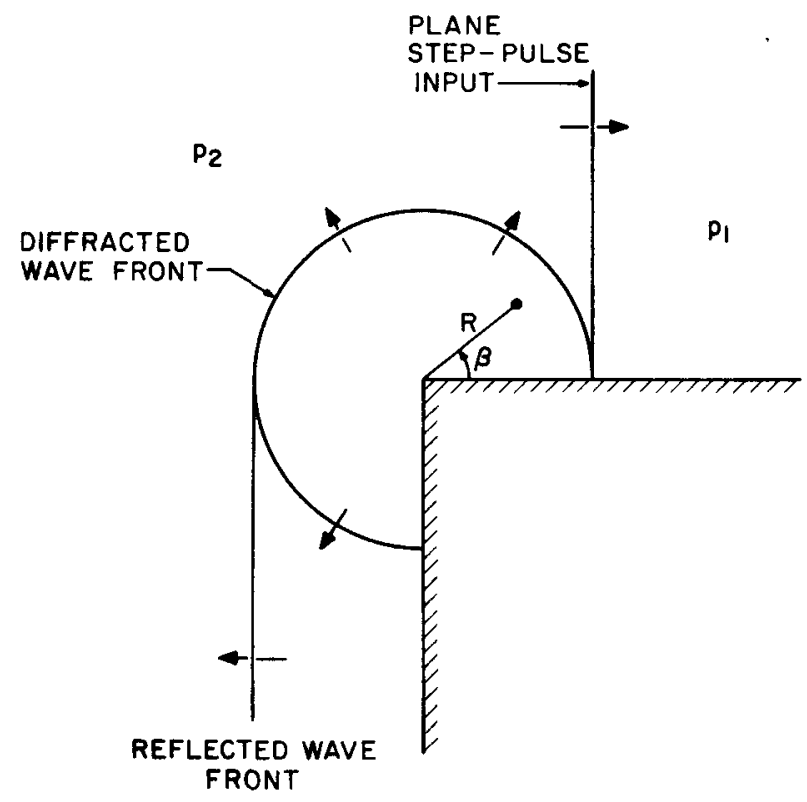

Fig. A.1. Compression Problem for $90^{\circ}$ Corner. ANL Neg. No. 113-77-544.

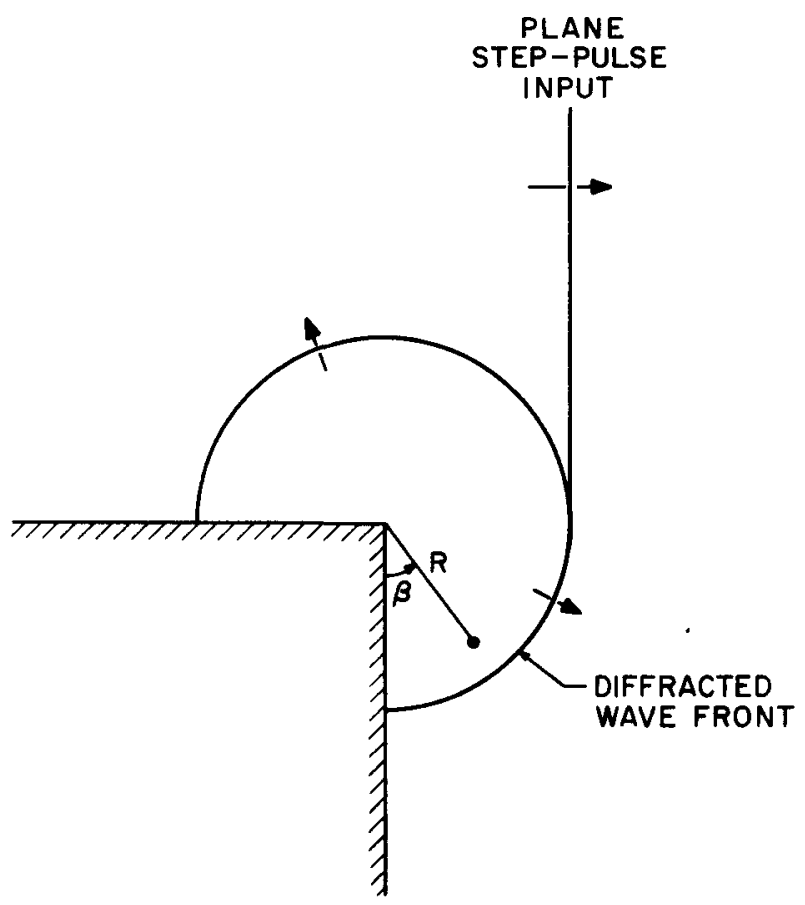

Fig. A.2. Expansion Problem for $90^{\circ}$ Corner. ANL Neg. No. 113-77-546. 


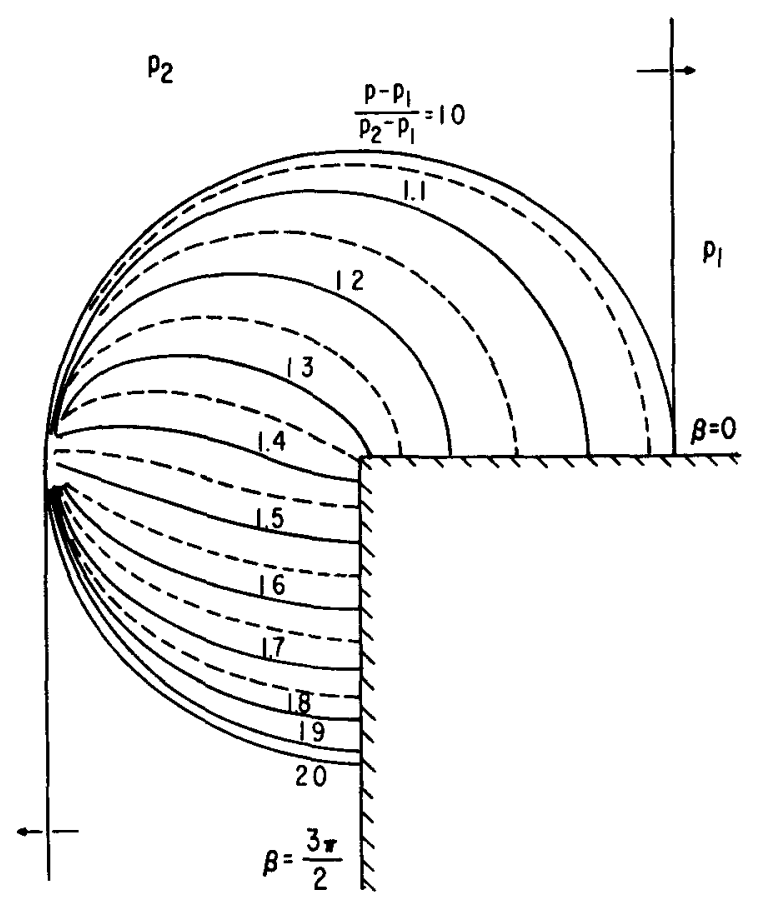

Fig. A.3. Exact Solution for Corner Compression. ANL Neg. No. 113-75-131 Rev. 1.

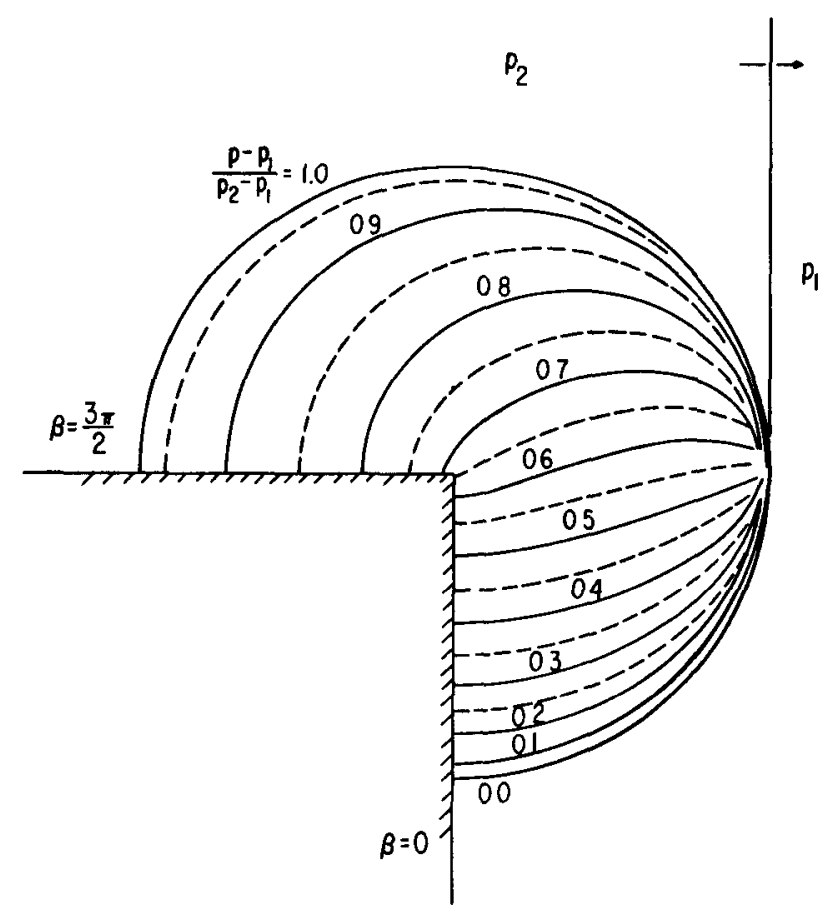

Fig. A.4. Exact Solution for Corner Expansion. ANL Neg. No. 113-75-132 Rev. 1.

Finally, the solution to the plane step pulse expanding into a sudden enlargement is constructed by a careful superposition of the occurring wave fronts. The individual wave fronts, as the step pulse propagates through the area transition are depicted in Fig. A.5, plotted with increasing time. The dark area is the area swept by wave fronts of curved waves diffracted from the corners. In this region, the superposition solution does not apply, since it is limited to plane-wave diffractions. The pressure histories at points $A, B, C, A^{\prime}, B^{\prime}$, and $C^{\prime}$ are constructed by the superposition technique, using the basic solution expressed by Eq. A.6 and plotted in Fig. A.4 for the expanding pulse. The result is shown in Fig. 7 where comparison is made with numerical results.
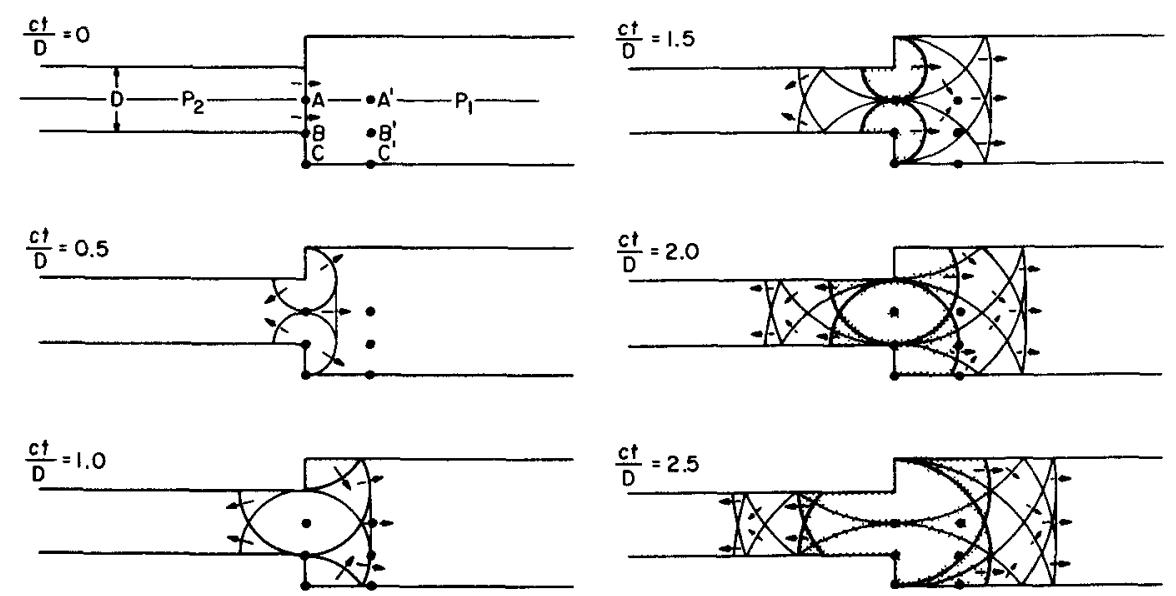

Fig. A.5. Individual Wave Fronts for Sudden Area Enlargement (Cartesian geometry). ANL Neg. No. 113-77-353 Rev. 1. 


\section{REFERENCES}

1. D. S. Butler, The Numerical Solution of Hyperbolic Systems of Partial Differential Equations in Three Independent Variables, Proc. R. Soc. London, Series A 255, 232-252 (1960).

2. D. J. Richardson, Solutions of Two-Dimensional Hydrodynomic Equations by the Method of Characteristics, Methods Comput. Phys. 3, B. Alder, S. Fernback, and M. Rotenberg, eds., Academic Press, New York, pp. 295-318 (1964).

3. Y. W. Shin and R. A. Valentin, Numerical Analysis of Fluid-Hormer Waves by the Method of Characteristics, J. Comput. Phys. 20, 220-237 (1976).

4. R. Sauer, Differenzenverfahren fur hyperbolische Anfangswert-probleme bei mehr als zwei unabhängigen Veränderlichen mit Hilfe von Nebencharakteristiken, Num. Math. 5, 55-67 (1963).

5. Y. W. Shin and C. A. Kot, Two-dimensional Fluid-hommer Analysis by the Method of Characteristics, ANL-75-21 (May 1975).

6. R. Courant, K. Friedrichs, and H. Lewy, Uber die Partielzen Differenzialgleichungen der Mathematischen Physik, Math. Ann. 100, 32-74 (1928).

7. J. B. Keller and A. Blank, Diffraction and Reflection of Pulses by Wedges and Comers, Commun. Pure Appl. Math. 4, 75-94 (1951).

8. A. Busemann, Infinitesimal Conical Supersonic Flow, Schr. Deutsch. Akad. Luftfahrtforschung $7 B(3), 105-122$ (1953).

9. D. R. White and W. Bleakney, Shock Loading of Rectangular Structure, Technical Report II-11, Dept. of Physics, Princeton University (1952).

10. J. B. Keller, Diffraction of a Shock or an Electromagnetic Pulse by a Right-AngZed Wedge, J. App1. Phys. 23, 1267-1268 (1952). 
Internal:
J. A. Kyger
C. K. Youngdahl
A. Amorosi
C. A. Kot
R. Avery
H. C. Lin
L. Burris
B. J. Hsieh
D. W. Cissel
G. F. Berry
S. A. Davis
J. Daley
B. R. T. Frost
W. T. Sha
D. C. Rardin
T. Bump
R. G. Staker
B. L. Boers
R. J. Teunis
W. L. Chen
C. E. Till
Y. Chang
R. S. Zeno
C. Wang
H. K. Fauske
P. S. Chopra
S. Fistedis
K. Kuczen
J. F. Marchaterre
H. U. Ahmed
H. O. Monson
A. B. Krisciunas
Y. W. Shin (30)
R. A. Valentin (10)
ANL Contract Copy
G. S. Rosenberg (2)
ANL Libraries (5)
TIS Files (6)

\section{External:}

DOE-TIC, for distribution per UC-79h (274)

Manager, Chicago Operations Office

Chief, Chicago Patent Group

Director, DOE-RRT (2)

Director, Reactor Programs Div., CH

Director, CH-INEL

President, Argonne Universities Association

Components Technology Division Review Committee:

J. W. Dally, U. Maryland

W. E. Kessler, Commonwealth As sociates

N. C. Rasmussen, Massachusetts Inst. Technology

M. A. Schultz, Pennsylvania State U.

A. Sesonske, Purdue U.

H. Thielsch, ITT Grinnell Corp.

N. J. Palladino, Pennsylvania State U. 